\title{
Wie Naturmärchen und partizipative Medienarbeit Bildung für nachhaltige Entwicklung vermitteln
}

In einem Aufnahmestudio auf Schloss Hundisburg bei Magdeburg erhält eine Gruppe von Kindern im Alter zwischen 10 und 12 Jahren vom Toningenieur endgültige Anweisungen für eine Hörspielaufnahme des Märchens „Kalif Storch“1 von Wilhelm Hauff. Die Aufnahme ist Teil eines neuen Projektdesigns der Nichtregierungsorganisation „Explority“2, welches Bildung für nachhaltige Entwicklung mit Media Advocacy ${ }^{3}$ zu Kinderrechten und nachhaltiger Entwicklung kombiniert und dabei mittels Märchen Kinder ins Zentrum eines schulfächerübergreifenden Konzepts zur Kompetenzförderung, Wissensvermittlung und Meinungsführerschaft fördert. Unter dem Programmtitel Young Voices for Sustainable Development ${ }^{4}$ werden Kinder zu Anwälten nachhaltiger Entwicklung, indem sie mit ihren eigenen Stimmen unter anderem das Bewusstsein für gegenwärtige Bedrohungen für die biologischer Vielfalt auf unserem Planeten schärfen. Mit dem märchenbasierten Ansatz „Nawa \& Bubo in Mittelelbien“ wird die jüngere Zielgruppe der Grundschulkinder an das Thema globale Entwicklung herangeführt. Die verwendeten Naturmärchen erfüllen dabei multiple Zwecke: a) Nutzung von Kultur zur Bewusstseinsschärfung zu Natur, b) Wissensvermittlung zu biologischer Vielfalt und globalen Herausforderungen zum Schutz dieser, c) der zielgruppenaffinen Ansprache und d) als Vehikel eines intergenerationellen Dialogs zur Bedeutung von Natur- und Klimaschutz für Grundschulkinder und ihren Familien.

Bei der Entwicklung und Umsetzung des Projektdesigns bezieht sich Explority auf ein diverses Policy Environment entwicklungspolitischer Bildung und nachhaltiger Entwicklung. Mit dem „Orientierungsrahmen für den Lernbereich Globale Entwicklung "5 haben die Kultusministerkonferenz (KMK) in Zusammenarbeit mit dem Bundesministerium für wirtschaftliche Zusammenarbeit und Entwicklung (BMZ) Modelle für ein fächerübergreifendes Konzept zur Vermittlung von globaler Entwicklung für die Zielgruppen Grundschule und Sekundarstufe I vorgelegt. Explority bezieht sich hier auf die dort einerseits vermittelten Strategien zur Bewusstseinsschärfung und Wertehaltungen sowie den andererseits den vermittelten Kernkompetenzen im fächerübergreifenden Lernbereich Globale Entwicklung. Für die Umsetzung der Media-Advocacy-Strategien finden sich Bezüge zu den Globalen Nachhaltigkeitszielen und zur Agenda 2030 der Vereinten Nationen (besonders zu Ziel 15, Biologische Vielfalt/Leben an Land) ${ }^{6}$, mit dem Ausüben des Rechts auf Meinungs- und Informationsfreiheit laut Artikel 13 der Kinderrechtskonvention ${ }^{7}$ sowie mit „Stärkung und Mobilisierung der Jugend" des Handlungsfeldes 4 der UNESCO-BNE-Strategie zu prioritären Handlungsfeldern in der BNE-Vermittlung.

In unserem vorliegenden Beispiel führt das Märchen Kalif Storch die Kinder in die Thematik Zugvögel ein und sensibilisiert sie für ihr regionales Ökosystem, in dem Störche leben, Sachsen-Anhalt und Sudan in Afrika. Die anfängliche Inszenierung des Märchens stellt bei dem von Explority entwickelten Projektdesign den wichtigen Aktivierungsgedanken von Media Advocacy dar. Im Rahmen einer Projektwoche erfuhren die
Grundschüler/-innen Hintergrundwissen zu den jeweiligen Lebensräumen des Storches und welche Bedeutung diese edlen Vögel für ihr Ökosystem haben. Im Anschluss ging es für die Kinder im Rahmen einer von Vogelexperten geführten Exkursion in den Naturpark Drömling, wo neben Weiß- auch Schwarzstörche im sog. „Land der tausend Seen“ ihre Heimat finden. Nebenbei sammelten die Kinder Naturgegenstände wie auch zivilisatorischen Müll, um den Lebensraum abzubilden. Zudem hatten die Kinder den Auftrag, mittels hochempfindlicher Mikrofone Geräusche sowie mit Fotokameras Fotos aus dem Lebensraum aufzunehmen, welche später in der Hörspielaufnahme bzw. Multimediaausstellung wiederzufinden waren. Sämtliche von den Kindern produzierten Medieninhalte der Kinder wurden schließlich zusammen mit den gesammelten Naturgegenständen, Informationen zum Ökosystem und den Umweltbotschaften der Kinder im Rahmen einer Multimediaausstellung präsentiert, in der sie der Öffentlichkeit ihre Forderungen nach Verhaltensänderungen zum Schutz regionaler Ökosysteme präsentierten.

Explority nutzt die Vorstellungskraft jüngerer Schulkinder, um ihnen zu ermöglichen, nicht nur globale Herausforderungen für die Natur zu verstehen, sondern auch Empathie und ein Mandat für sozialen Wandel anzuwenden. Nach dem erfolgreichen Pilotprojekt in Sachsen-Anhalt, in dem 10 Schulklassen 10 verschiedene Naturthemen im Kontext nachhaltiger Entwicklung erarbeiteten, und der Publikation einer Heftreihe mit 10 Ausgabenverschiedenen Natur- und Bildungsthemen ${ }^{8}$, hat Explority in 2020 ein neues dreijähriges Programm für Nawa \& Bubo gestartet. Dabei werden kontinentale Fokusthemen aus Afrika, Asien und Lateinamerika zu nachhaltiger Entwicklung mittels einheimischer Märchen von den Kindern in Deutschland auch mit Hilfe virtueller Begegnungen mit Gleichaltrigen auf der Südhalbkugel erarbeitet. Weitere Informationen unter: https://explority.org

\section{Anmerkungen}

1 Kalif Storch aus der Rahmenerzählung: Die Karawane, Wilhelm Hauff, Märchenalmanach (1826), Aufruf am 14.10.2020 https://www.projekt-gutenberg.org/hauff/ alma1826/chap003.html

2 Explority-Zentrum für nachhaltige Bildung und Kompetenzforschung e.V., Aufruf am 14.10.2020 https://explority.org

3 "Advocacy“ ist ein englischsprachiger Fachbegriff, der sich ins Deutsche als themenanwaltschaftliche Vertretung übersetzen lässt. Media Advocacy bedeutet die Umsetzung von Advocacy Strategien mittels Medien

4 Young Voices for Sustainable Development ist ein Programm zur Förderung demokratischer Rechte und Kinderrechte, für welche Explority die Internetplattform https:// sdgyoungvoices.explority.org/ unterhält.

5 Aufruf am 14.10.2020 https://www.globaleslernen.de/sites/default/files/files/linkelements/orientierungsrahmen_fuer_den_lernbereich_globale_entwicklung_barr ierefrei.pdf

6 Aufruf am 14.10.2020 https://17ziele.de/ziele/15.html

7 Aufruf am 14.10.2020 https://www.unicef.de/informieren/ueber-uns/fuer-kinder rechte/un-kinderrechtskonvention

8 Aufruf am 14.10.2020, vgl. https://sdgyoungvoices.explority.org/oer/nawa-bubo

Wolfgang J. Fischer Explority - Zentrum für nachhaltige Bildung und Kompetenzforschung e.V. doi.org/10.31244/zep.2020.04.07 